\title{
Novel method of severe plastic deformation - continuous closed die forging: CP aluminum case study
}

\author{
Alexander P. Zhilyaev ${ }^{1,2, a}$ Sandra Rodriguez ${ }^{3,4, b}$ Jessica Calvo ${ }^{4, c}$ \\ José María Cabrera ${ }^{4, d^{*}}$
}

\author{
${ }^{1}$ Laboratory of Mechanics of Gradient Nanomaterials, Nosov Magnitogorsk State Technical \\ University, Magnitogorsk, 455000, Russia \\ ${ }^{2}$ Institute for Metals Superplasticity Problems, Khalturina 39, Ufa, 450001, Russia \\ ${ }^{3}$ Universidad Autónoma de San Luis Potosí, Facultad de Ingeniería 78290, S.L.P., México \\ ${ }^{4}$ Departamento de Ciencia de los Materiales e Ingeniería Metalúrgica, EEBE - Universitat \\ Politècnica de Catalunya, Barcelona, 08019, Spain
}

aalex.zhilyaev@hotmail.com, bsandyreyna@uaslp.mx,
cjessica.calvo@upc.edu; djose.maria.cabrera@upc.edu

Keywords: CCDF, UFG, EBSD, Mechanical properties and Microhardness.

\begin{abstract}
There is a large number of methods for severe plastic deformation (SPD). Multidirectional forging (MDF) is probably one of the most easily scalable for industrial application. In general, two main conditions need to be fulfilled for successful SPD processing: constant sample geometry and application of a quasi-hydrostatic pressure. The first condition is necessary for strain accumulation by repetitive deformation and the second one helps preventing cracking in the specimens with high accumulated strain. However, MDF is not providing quasihydrostatic condition in the processed sample. This paper reports a novel method for severe plastic deformation, namely continuous closed die forging (CCDF), which fulfils both requirements for the successful deformation of samples to a very high accumulated strain. Commercially pure aluminum (1050) was processed to a total strain of 24 by CCDF. After processing, the microstructure was refined down to a mean grain size of $0.78 \mu \mathrm{m}$. Tensile testing showed good mechanical properties: yield strength and ultimate tensile strength of the ultrafine-grained (UFG) aluminum were 180 and $226 \mathrm{MPa}$, respectively. Elongation to rupture was about 18\%. The microstructure, microhardness and grain boundary statistics are discussed with regard to the high mechanical properties of the UFG aluminum processed by this novel method.
\end{abstract}

\section{Introduction}

The microstructure plays an important role in the physical and mechanical properties of polycrystalline materials, being the grain size one of the most important characteristics. The relationship that exists between the yield stress, $\sigma_{Y}$, and the grain size, $d$, of polycrystalline materials can be expressed through the Hall-Petch relationship detailed in equation 1 [1], which indicates that the yield stress increases when reducing the grain size.

$$
\sigma_{y}=\sigma_{0}+k \cdot d^{-1 / 2}
$$

Therefore, obtaining materials with Ultra Fine Grain size (UFG), in the range between $100 \mathrm{~nm}$ and $1 \mu \mathrm{m}$, or even lower, has been of great interest for the scientific community in the last decades. If a process of Severe Plastic Deformation (SPD) is applied to a material, a UFG structure can be obtained with high angle grain boundaries. SPD processes introduce a great amount of strain in the material, therefore it must be performed at low temperatures in the presence of large hydrostatic pressures to avoid crack nucleation and propagation, which would compromise the integrity of the 
workpiece [2]. Currently there are several SPD processes that meet the above specifications and generate uniform microstructures throughout the volume of the piece, which is important to have stability in the mechanical properties and subsequent forming processes.

SPD processes, among the top-down methods, have been proven to be an effective and promising alternative for the mass production of UFG materials and present advantages when compared to the bottom-up approach based on nano-powder compaction, namely lack of porosity, reduced levels of impurities and industrially scalable in terms of the process times and required investment $[3,4,5]$. In addition to the most widely explored SPD processes, HPT (High Pressure Torsion) and ECAP (Equal Channel Angular Pressing), MDF (Multidirectional forging) can also be scaled to industrial production $[6,7,8]$. The basic principle of MDF is the repetitive application of compression to a material, varying the axis of application of the deformation in each pass. The redundant plastic deformation will accumulate after each pass, whether the deformation occurs at low or high temperature. The repetitive variation of the axis of application of the deformation is important for the refinement of the microstructure [9].

In this research work, a novel MDF method, named Continuous Closed Die Forging (CCDF), has been applied to a commercially pure aluminum (1050) impairing a total strain of 24 , leading to improved mechanical properties.

\section{Experimental Procedure}

Four bars of AW-Al-1050A with a diameter of $20 \mathrm{~mm}$ and a length of $100 \mathrm{~mm}$ were subjected to pre-annealing (a bar in this condition is used as a control sample). The heat treatment was applied to the samples in an HOBERSAL 12 PR / 300 oven. Once the oven reached $630{ }^{\circ} \mathrm{C}$, the pieces were placed inside and the temperature was maintained constant for 48 hours. This annealing was followed by a cooling at $66^{\circ} \mathrm{C} /$ hour until reaching $300^{\circ} \mathrm{C}$, temperature at which the material remained for 3 hours prior to a final furnace cooling to room temperature. This pre-annealing heat treatment has been reported to be effective in increasing the yield strength of 1050 aluminum alloy processed by ECAP [10].

The principle of CCDF processing is shown in Fig. 1. The matrix is composed of two dies which form a rhomboidal inner cross section. The samples, with an initial circular section of $20 \mathrm{~mm}$ in diameter, were placed in the cavity of the matrix, as shown in Fig. 1. In the " 1 st step" a closing load is applied to the dies trough a rod which is held for 10 seconds, forcing the sample to acquire the inner shape of the matrix. After the retraction of the rod, the dies are open and the sample piece is turned 90 degrees counterclockwise as represented in Fig. 1 as " 2 nd step". In this new position of the sample, a closing load is again applied and the material flows until it fills the space between the dies. The final shape of the samples after each deformation pass is rhomboidal with a major axis of less than $15 \mathrm{~mm}$ and a second axis which depends on the hardness of the material. Molybdenum disulfide lubricant is used in the process. The displacement speed of the rod was $5 \mathrm{~mm} / \mathrm{s}$ and the temperature was controlled using a thermocouple and a data acquisition system. Following this CCDF route, up to 24 passes could be applied to the samples before crack appearance.

After the CCDF, samples were extracted from the central part of the same cross section as fig 1 for metallografic obersvation. Samples were grinded and polished following standard procedures. Micro-indentation tests were carried out using Akashi-HO micro-hardness tester, with a Vickers four sided pyramidal indenter. Additionally, the mechanical properties were determined on cylindrical samples machined from the central longitudinal axis of the rod after CCDF processing using a universal machine INSTRON model 4507. In the tensile tests, a deformation speed of 0.001 $\mathrm{s}^{-1}$ and a preload of $10 \mathrm{~N}$ were used. Test was carried out until the rupture of the sample. 

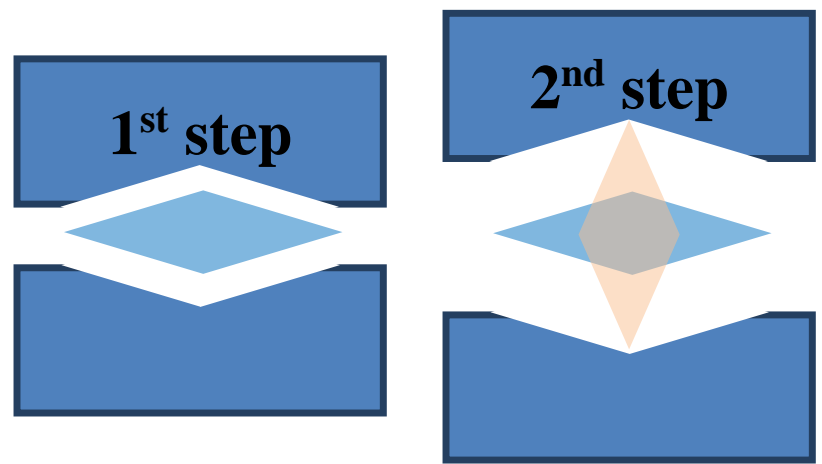

Fig. 1. Schematic of continuous closed die forging $(C C D F)$.

For further metallographic interpretation, sectioned samples were electropolished and the grain structures were recorded by orientation imaging microscopy (OIM) using the electron back scattered diffraction (EBSD) technique integrated in SEM brand JEOL, model JSM-5600 (controlled and analyzed using channel 5 software). The grain size slightly differed between the samples, and different area dimensions and step sizes were chosen to maximize the number of data points and still get a good statistical results. The statistical variation on grain size and misorientation angle, obtained by EBSD, were used for the interpretation of the mechanical results.

\section{Results and Discussion}

Fig. 2 (a, b, c and d) shows the evolution of the cross section of the sample after 8, 16 and 24 passes of CCDF. The initial shape of the specimens was circular and became rhombohedral after the first and subsequent deformation passes, since this is the inner shape of the matrix used. When observing in greater detail the images, the formation of a cross can be visualized caused by a strain concentration in the vertices of the matrix. However, it is important to highlight the high strain accumulated inside the piece in order to achieve 24 deformation passes without the presence of any visible crack. It must be pointed out that the microstructure was not fully homogeneous, as large amount of deformation was concentrated in the central part of the section, while the edges received less strain. Fig. 3 (a) shows the EBSD texture map of the sample processed by 24 passes of CCDF, which was the highest deformation applied following the novel SPD method. Fig. 3 (b) presents the grain boundary map of the same sample. In this image, the HABs (High Angle Boundaries) with misorientation angles larger than $15^{\circ}$ are represented as black lines, while the LABs (Low Angle Boundaries) with misorientation angles lower than $15^{\circ}$ are represented as green lines. The sample contains a mixture of elongated and equiaxed grains, and the fraction of HAB and LABs is $83.4 \%$ and $10.4 \%$, respectively.

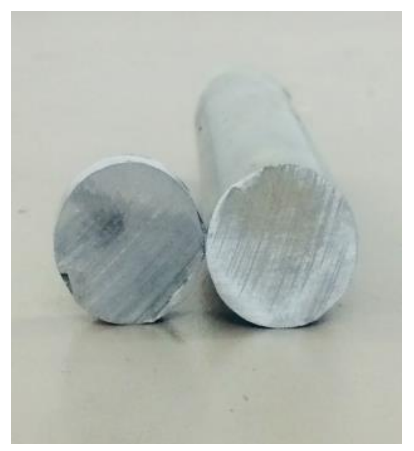

(a)

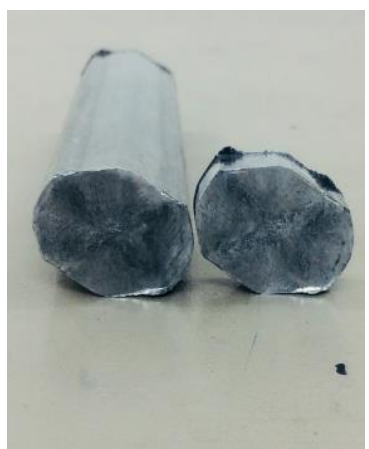

(b)

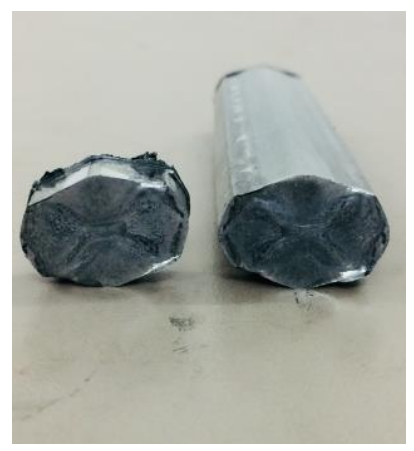

(c)

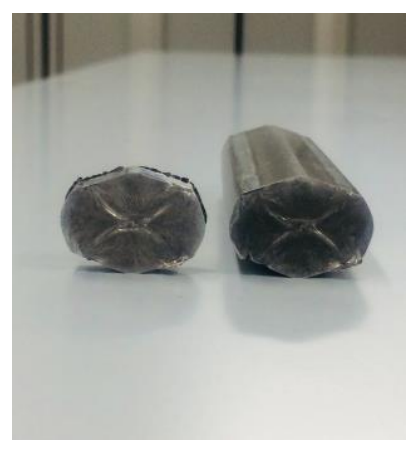

(d)

Fig. 2. Evolution of shape Al-1050 by CCDF (a) 0 Passes; (b) 8 Passes;

(c) 16 Passes and (d) 24 Passes. 
In Fig. 3 (c) complementary statistical information is provided on the grain size distribution taken from the EBSD analysis on the 24 CCDF passes sample. The commercial Al-1050 grain size is approximately $150 \mu \mathrm{m}$ [11]. The EBSD data reveals that the average grain size decreases to $0.78 \mu \mathrm{m}$ after 24 passes of CCDF on a pre-annealed sample. The distribution observed in Fig. 3 (c) corresponds to an UFG material.

Fig. 3 (d) displays the misorientation distribution of boundaries obtained from the EBSD data. As stated, the distribution is of a bimodal type with two different modes, low and high angles, as traditionally reported in the literature for highly deformed samples [12]. Chang et al. reported that the dislocation density inside the grains of a 1050 aluminum alloy decreases with an increase of the strain, and most of the grains eventually became free of dislocations, due to continuous dynamic recrystallization during the SPD processes after large deformations [13].

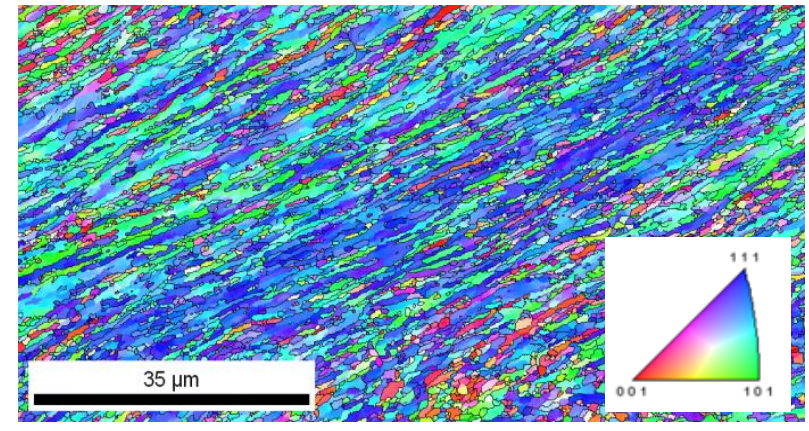

a)

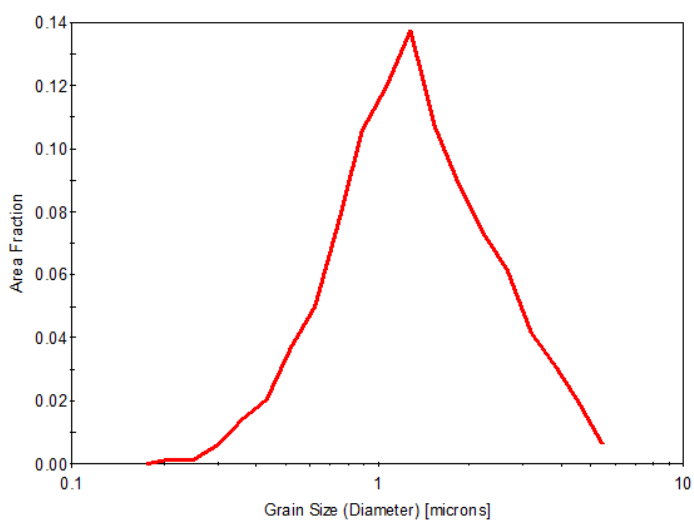

c)

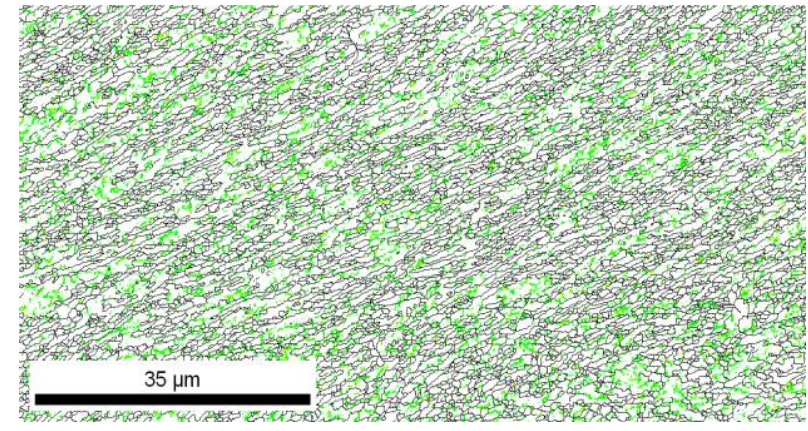

b)

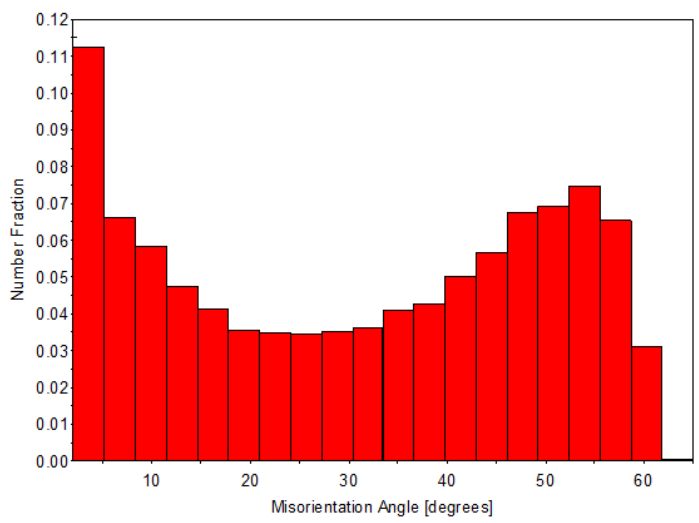

d)

Fig. 3. EBSD results: (a) Texture and (b) Grain boundary maps (black: HAGB, green:LAGB); (c) grain size distribution and (d) grain boundary misorientation distribution of Al 1050 processed to 24 passes by $C C D F$.

Fig. 4. shows the Stress-Strain curves of samples machined from bars subjected to 16 and 24 deformation passes, with and without control of the loading time, together with the reference material, only subjected to the pre-annealing heat treatment. The control of the loading time (10 s according to the "Experimental Procedure" section, was introduced in the processing route in order to assure the correct flow of the material and filling of the dies. From the load versus elongation diagram, the properties of $0.2 \%$ YS (Yield Strength), UTS (Ultimate Tensile Strength), uniform elongation, and elongation to rupture were obtained and are summarized in Table 2. The materials processed by CCDF showed much higher YS and UTS values, compared to the Al-1050 preannealed material. 


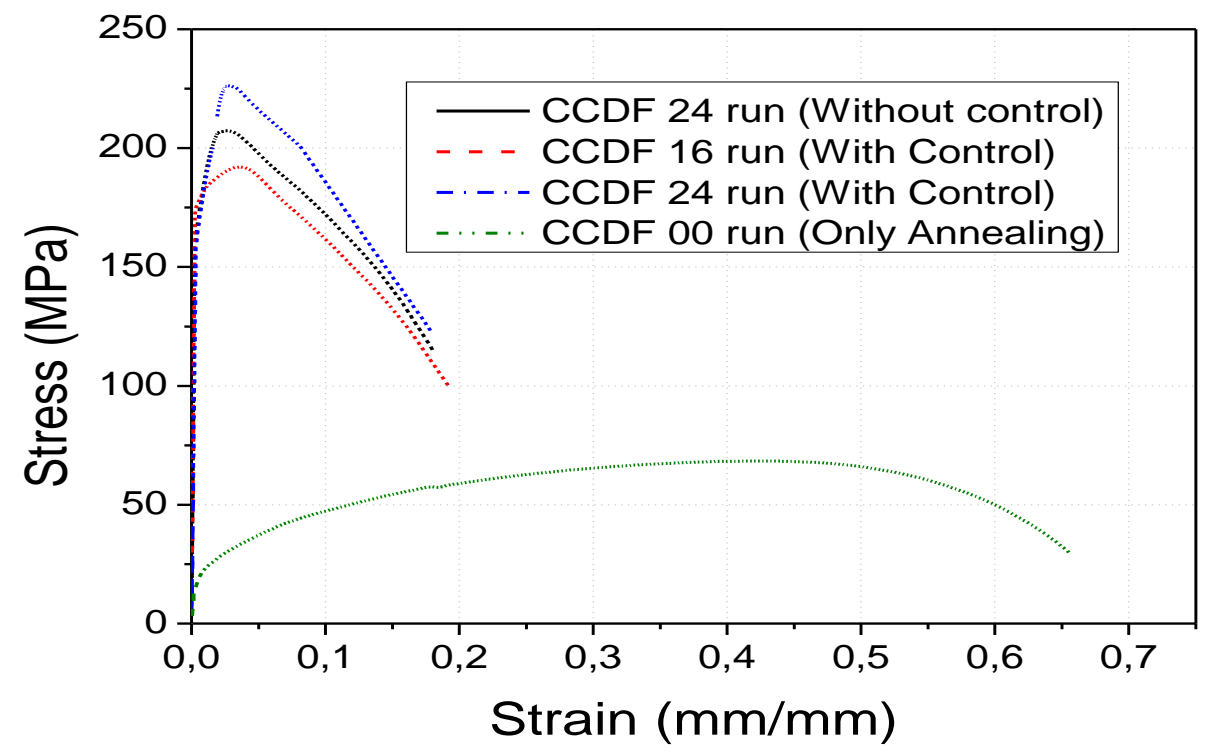

Fig.4. Stress-strain curves of Al-1050 by CCDF (With 16 and 24 passes).

Table 1. Mechanical Properties of Al-1050 by CCDF.

\begin{tabular}{|l|l|l|l|l|}
\hline Specimen & $\begin{array}{l}\text { YS } \\
\mathbf{0 . 2 \%}(\mathbf{M P a})\end{array}$ & $\begin{array}{l}\text { UTS } \\
\text { (MPa) }\end{array}$ & $\begin{array}{l}\text { Elongation } \\
\text { Uniform (\%) }\end{array}$ & $\begin{array}{l}\text { Elongation } \\
\text { rupture (\%) }\end{array}$ \\
\hline 00 run (only Annealing) & 20 & 70 & 44 & 66 \\
\hline 16 run (With control) & 170 & 190 & 4 & 19 \\
\hline 24 run (Without control) & 170 & 208 & 3 & 18 \\
\hline 24 run (With control) & 180 & 226 & 3 & 18 \\
\hline
\end{tabular}

The increase in the strength is attributed to i) the increased dislocation density, which promote the generation of subgrain walls and ii) the grain size refinement due to continuos dynamic recrystallization. It is noteworthy the increase of the YS from $20 \mathrm{MPa}$ to $180 \mathrm{MPa}$ after 24 passes of CCDF. It can also be noted that the loading time control in the CCDF process can positively affect the increase of the mechanical properties. Although the percentage in uniform elongation and elongation to rupture with the increase in CCDF passes was reduced, these values are acceptable.

The relationship between the Vickers indentation hardness (HV) and yield strength YS for the ultrafine grained materials produced by SPD process can be described based on equation (2):

$$
H_{V}=\left(Y S_{M P a} / 3\right) \cdot(0.1)^{n *}
$$

where $n^{*}$ is the modified work-hardening exponent ( 0.1) [14]. The hardness of the pre-annealed Al-1050 alloy has a value of $28 \mathrm{HV}$. The average hardness value after 24 passes of CCDF is $48 \mathrm{HV}$. Applying equation 2, the value is $47.6 \mathrm{HV}$ very close to that obtained in the laboratory. It is also observed that some deformation fields are generated due to the anisotropy which is probably a result of the elongated grain structures observed in Fig. 3 (a), indicating that the indentation resistance is greater along the fine dimension of the grains, perpendicular to the forging direction [15]. However, there is enough evidence which indicates that the refinement of grain observed in the grain size distribution shown in Fig. 3 (c) contributes positively to the increase of the mechanical properties. 


\section{Conclusions}

Through a novel method of severe plastic deformation, called continuous close die forging CCDF, an ultrafine grain size was obtained, with an average value of $0.78 \mu \mathrm{m}$ in a commercial Al-1050. The work pieces after 16 and 24 passes do not present visible fracture in the whole volume, maintaining their rhombohedric form from the first pass. The refinement of grain in the samples processed by CCDF makes possible to explain the behavior of the material by presenting superior mechanical properties (YS, UTS and HV) to the commercial material.

\section{Acknowledgments}

A.P. Zhilyaev gratefully acknowledges financial support from the Ministry of Education and Science of the Russian Federation (Grant 14.Z50.31.0043).

\section{References}

[1] R. Valiev, A. Zhilyaev, T. Langdon. Hoboken, New Jersey : John Wiley and Sons. Inc., 2014.

[2] R. Valiev, R. Islamgaliev, I. Alexandrov. Prog. Mater. Sci. 45 (2000) 103-189.

[3] I. Sabirov, Yu. Murashkin, R. Z. Valiev. Mater. Sci. Eng. A. 560 (2013) 1-24.

[4] R.Z. Valiev, R.K. Islamgaliev, I.V. Alexandrov. Prog. Mater. Sci. 45 (2000) 103-189.

[5] M. Dao, L. Lu, R.J. Asaro, J. T. M. DeHosson, E. Ma. Acta. Mater. 55 (2007) 4041-4065.

[6] R.Z.Valiev, T.G. Langdon, Prog. Mater. Sci. 51 (2006) 881-981.

[7] A.P. Zhilyaev, T.G. Langdon, Prog.Mater.Sci.53 (2008) 893-979.

[8] O.R. Valiahmetov, R.M. Galeyev, G.A.Salishchev, FizMetall. Metall oved. 70 (1990) 204-206.

[9] M. Montazeri-Pour, M.H. Parsa, H.R. Jafarian, S. Taieban. Mater. Sci. and Eng. A. 639 (2015) 705-716.

[10] Y. Cao, L.He, Y . Zhou, P. Wang, J. Cui. Mater. Sci. and Eng. A. 674 (2016) 193-202.

[11] A. Mustafa, Abdulstaar, El-Danaf, S. Waluyo. Mater. Sci. Eng. A. 565 (2013) 351-358.

[12] N. Kamikawa, X. Huang, N. Tsuji, N. Hansen. Acta Mater. 57 (2009) 4198-4208.

[13] C.P. Chang,P. L. Sun, P. W. Kao. Acta. Mater. 48 (2000) 3377-3385.

[14] N. Hansen, Hall-Petch. Scr. Mater. 51 (8)(2004) 801-806.

[15] F. Khodabakhshi, A.P. Gerlich. Materials Characterization.136 (2018) 229-239.

[16] M. Naderi, M. Peterlechner. Mater. Sci. Eng. A 708 (2017) 171-180 